\title{
Complementary Sequences for ISI Channel Estimation
}

\author{
Predrag Spasojević, Member, IEEE, and Costas N. Georghiades, Fellow, IEEE
}

\begin{abstract}
A merit factor based on the sequence autocorrelation function, whose minimization leads to the reduction in the Crámer-Rao lower bound (CRLB) for the variance of "two-sided" intersymbol interference (ISI) channel estimation is introduced. Pairs of binary pilot symbol sequences (a preamble and a postamble) for channel estimation are jointly designed to minimize this merit factor. Given that the number of channel taps is $L$ and the length of a pilot symbol sequence is $(N+L-1)$, where $N \geq L$, we distinguish between the case when $N$ is even and the case when it is odd. For even $N$, we show that complementary sequences not only minimize the merit factor, but also the CRLB. For a subset of odd $N$ we construct almost-complementary periodic sequence pairs that minimize the merit factor. The optimal pilot symbol block signaling requires alternating between two (in most cases) different binary sequences that form the merit-minimizing pair.
\end{abstract}

Index Terms-Autocorrelation merit function, complementary sequences, intersymbol interference (ISI) channels, pilot symbols.

\section{INTRODUCTION}

D IGITAL communications over mobile channels can be severely degraded due to unknown time-variant fading of the received signal. It is a common approach to periodically insert known symbols in order to reliably estimate the channel parameters prior to detection (see, e.g., [1], for flat fading channels). In the case of time-variant multipath fading channels, where the path delay spread is on the order of several symbols or larger, pilot symbol blocks that span the channel memory need to be inserted (see, e.g., [2]). In deriving optimal, or some decision-feedback detection and channel estimation algorithms, the signal is frequently assumed to be quasistatic in an interval encompassing a number of transmitted symbols (see, e.g., [2] (and references therein) and [3]).

As in [4] for pilot symbols, [5] has employed both pilot symbol blocks (preamble and postamble) that frame a block of data (as seen in Fig. 1) for estimation of the (quasi-static) channel coefficients pertaining to a particular data block. This approach we term "two-sided" channel estimation. Here, we introduce optimal binary sequences for two-sided channel estimation. Previously, only sequences for one-sided channel estimation have been considered (for training using binary

Manuscript received July 9, 1999; revised May 27, 2000. This work was supported in part by the National Science Foundation under Grant NCR-9314221.

P. Spasojević is with WINLAB, Electrical and Computer Engineering Department, Rutgers University, Piscataway, NJ 08854 USA (e-mail: spasojev@winlab.rutgers.edu).

C. N. Georghiades is with the Department of Electrical Engineering, Texas A\&M University, College Station, TX 77843 USA (e-mail: georghia @ ee.tamu.edu).

Communicated by M. Honig, Associate Editor for Communications.

Publisher Item Identifier S 0018-9448(01)01339-6. sequences, see [6]-[9] and, more recently, [10]). As will be shown, the constructed optimal sequences will require that the two pilot symbol blocks framing a data block differ in most cases and, therefore, the optimal signaling will require alternating periodically inserted training blocks.

A merit factor based on the periodic autocorrelation function (PACF) of the two binary pilot sequences framing a data block is introduced. The minimization of this merit factor will lead to the reduction in the channel estimation Crámer-Rao lower bound (CRLB). Previous merit factors that have been introduced attempt a reduction in the ratio of the autocorrelation function energy at nonzero shifts to its value at the zero shift (see, e.g., [11]). The proposed merit factor has a mini-max form (similar to the criterion derived for pulse-position modulation (PPM) sequences in [12] and to the phase optimization criterion for peak-to-average power reduction in orthogonal frequency-domain multiplexing (OFDM) in [13]). The selection criterion is based on an upper bound for the channel estimation variance. This bound is derived using the Gerschgorin discs pertaining to the eigenvalues of the two-sided pilot symbol matrix. The two-sided pilot symbol matrix is formed by summing the autocorrelation matrices of the two pilot symbol sequences framing the data-block. The optimal selection requires minimization of the maximum Gerschgorin disc radius. Unlike the criteria employed in [8] and [10], which are used mainly for computer-aided search of optimal sequences, the merit factor derived here aids in analytical construction of optimal sequences based on previously known binary sequences having good periodic autocorrelation properties.

The number of symbols per pilot symbol block is assumed to be $(N+L-1)$, where $L$ is the number of channel taps. For the case when the channel estimate is based on pilot symbol blocks with even $N$, we suggest complementary sequences ([14], [15]) as pilot symbol sequence pairs that not only minimize the merit factor, but also achieve the minimum estimation CRLB. Complementary sequences have previously been employed for channel characterization in [16] due to their good autocorrelation characteristics. ${ }^{1}$ Also, authors in [17] have suggested choosing one sequence from a complementary pair as a good choice for estimation of long channels when an optimal single-sided pilot sequence can not be easily found. Nevertheless, our paper is a first study that derives the complementary sequence pairs as optimal sequences for two-sided channel estimation. Furthermore, for a subset of odd $N$, we have constructed merit-minimizing sequence pairs based on a sufficient condition satisfied by their periodic autocorrelation

\footnotetext{
${ }^{1}$ The authors would like to thank the anonymous reviewers for suggesting [13] and [16].
} 


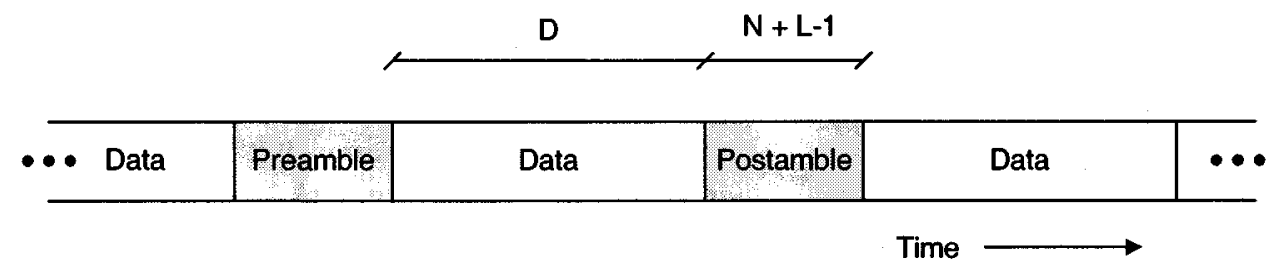

Fig. 1. Illustration of two-sided pilot symbol block insertion.

functions. Introduced sequence pairs are termed almost-complementary periodic sequences. For a different subset of odd $N$ construction of sequence pairs that have a "good" merit factor is also described.

Section II describes the signal model and the problem of "two-sided" channel estimation. Section III discusses the sequence selection criterion. Section IV solves the two-sided estimation problem by suggesting optimal pilot symbol sequence pairs. Analysis of Section $\mathrm{V}$ addresses proximity to the CRLB of the estimation variance, detection performance improvement when using optimal sequence pairs, and the effects of an increase in the pilot symbol length.

\section{Signal ModEL AND Estimation VARIANCE}

For simplicity, we assume that the received signal is a symbolspaced sequence (the oversampled case can be treated in a similar manner). In this case, a normalized block of received samples over which the channel is (quasi-)static can be expressed as follows (see, e.g., [5]):

$$
r=A h+n
$$

$\boldsymbol{r}$ is a received sample $(D+2 N+L-1) \times 1$ vector; $\boldsymbol{n}$ is a vector of a discrete white complex circular Gaussian noise process with a two-sided power spectral density (PSD) $N_{0} / E_{s}$, where $E_{s}$ is the symbol energy; $\boldsymbol{h}$ is a $L \times 1$ vector of coefficients of a linear (quasi-) time-invariant complex channel.

$\boldsymbol{A}$ is a Toeplitz matrix corresponding to the transmitted sequence of symbols whose elements are either +1 or -1 ; The $j$ th column of $A$ is a snapshot of the transmitted symbol sequence shifted by $L-j$ symbols. We will assume that the transmitted sequence has the form given in Fig. 1 where any frame of data symbols of length $D$ is framed with two $(N+L-1)$ pilot symbols, a preamble and a postamble. Furthermore, it will be assumed that the snapshot has been taken in such a manner that the first and the last row of the matrix $\boldsymbol{A}$ hold, respectively, the first and the last $L$ symbols of the preamble and the postamble. In this case, the matrix $A$ has the following form:

$$
\boldsymbol{A}=\left[\begin{array}{c}
\boldsymbol{P}_{1} \\
\boldsymbol{D} \\
\boldsymbol{P}_{2}
\end{array}\right]
$$

where $P_{1}$ and $P_{2}$ are $N$ by $L$ pilot symbol submatrices and $D$ is a $D+L-1$ by $L$ data submatrix. The pilot symbol submatrices are Toeplitz matrices consisting of only preamble and postamble symbol sequences of length $(N+L-1)$ and no data symbols.
$D$ is a $D+L-1$ by $L$ submatrix that holds all the data symbols and $2(L-1)$ pilot symbols of which one half pertains to each pilot symbol block. The received signal and the noise vector can be decomposed into subvectors that correspond to submatrices of $\boldsymbol{A}$ as $\boldsymbol{r}=\left[\boldsymbol{r}_{1}^{T} \boldsymbol{r}_{d}^{T} \boldsymbol{r}_{2}^{T}\right]^{T}$ and $\boldsymbol{n}=\left[\boldsymbol{n}_{1}^{T} \boldsymbol{n}_{d}^{T} \boldsymbol{n}_{2}^{T}\right]^{T}$ such that, e.g., $\boldsymbol{r}_{1}=\boldsymbol{P}_{1} \boldsymbol{h}+\boldsymbol{n}_{1}$.

In this paper, unless otherwise specified, even periodic will be termed any sequence with a period $N$, and odd-periodic will be termed a sequence $\boldsymbol{u}^{\circ}$ with a period $2 N$ such that $u_{i+N}^{O}=-u_{i}^{O}$, for any $i$. When the pilot-symbol blocks are subsequences of either odd- or even-periodic sequences, a pilot symbol submatrix is completely defined by one of its columns.

First, we briefly analyze the situation in which the channel estimate is provided only by the preamble. It is easy to show that the maximum-likelihood (ML) (in this case, the same as the least-squares) estimate of the channel has a variance that achieves the Crámer-Rao bound

$$
\frac{N_{0}}{E_{s}} \operatorname{tr}\left\{\left(\boldsymbol{P}_{1}^{H} \boldsymbol{P}_{1}\right)^{-1}\right\}
$$

where $H$ is the Hermitian transpose operator. Clearly, the estimate variance will be unbounded if the data matrix is "short," i.e., for $N<L$. In the following, we assume that the pilot symbol blocks are of a length for which the one-sided channel estimation can have a bounded variance. That is, any pilot symbol block is of length $(N+L-1) \geq 2 L-1$, i.e., $N \geq L$. Furthermore, we observe that the minimum attainable variance of the "one-sided" channel estimate is $\frac{N_{0}}{E_{s}} \frac{L}{N}$. If one requires $N=L$, it is known (see, e.g., [8]) that $\frac{N_{0}}{E_{s}} \frac{L}{N}$ can only be attained for $N \in\{1,2,4\}$. For $N=4$ the minimizing binary sequence is $[1,-1,-1,-1]$ and for $N=2^{L}+L$ extended $m$-sequences can be used for attaining this lower bound [8].

For a subset of even $N \geq L$, we will construct sequence pairs that allow for the minimum achievable estimate variance of

$$
\frac{N_{0}}{E_{s}} \frac{L}{2 N}
$$

in the case of "two-sided" channel estimation. For a subset of odd $N$, sequence pairs will be constructed for which the twosided channel estimation CRLB is close to this absolute lower limit.

Two-sided ML channel estimation is based on the "two-sided" pilot-symbol matrix

$$
P=\left[\begin{array}{l}
\boldsymbol{P}_{1} \\
\boldsymbol{P}_{2}
\end{array}\right]
$$


of size $2 N \times L . P$ is not a Toeplitz matrix, on the other hand, $\boldsymbol{P}_{1}$ and $\boldsymbol{P}_{2}$ are unequal Toeplitz matrices in the general case. The variance of the ML channel estimate is now

$$
\frac{N_{0}}{E_{s}} \operatorname{tr}\left\{\boldsymbol{R}^{-1}\right\}
$$

where

$$
\boldsymbol{R}=\boldsymbol{P}^{H} \boldsymbol{P}=\boldsymbol{P}_{1}^{H} \boldsymbol{P}_{1}+\boldsymbol{P}_{2}^{H} \boldsymbol{P}_{2}
$$

is the two-sided Grammian matrix of the column vectors of $\boldsymbol{P}$.

\section{VARIANCE BoundS AND THE MERIT FACTOR}

Our primary goal is to design Toeplitz matrices $\boldsymbol{P}_{1}$ and $\boldsymbol{P}_{2}$ with elements from the set $\{+1,-1\}$ that minimize $\operatorname{tr}\left\{\boldsymbol{R}^{-1}\right\}$ given $N$ and $L$. This problem can be solved using computeraided methods, but this approach is cumbersome and can be impossible for some sequence lengths. Our approach is to obtain a related merit factor (selection criterion) whose minimization will allow for construction of sequence pairs with minimum or, at least, low $\operatorname{tr}\left\{\boldsymbol{R}^{-1}\right\}$.

For a nonsingular $\boldsymbol{R}, \operatorname{tr}\left\{\boldsymbol{R}^{-1}\right\}=\sum_{i=1}^{L} 1 / \lambda_{i}$, where $\lambda_{i}$ are the eigenvalues of $\boldsymbol{R}$. Thus, we can obtain the following bounds on CRLB:

$$
\frac{N_{0}}{E_{s}} \frac{L}{2 N} \leq \frac{N_{0}}{E_{s}} \operatorname{tr}\left\{\boldsymbol{R}^{-1}\right\} \leq \frac{N_{0}}{E_{s}} \frac{L}{\lambda_{\min }}
$$

where $\lambda_{\min }=\min _{i}\left\{\lambda_{i}\right\}$. The lower bound is achieved iff all the off-diagonal elements $\rho_{i j}$ of $\boldsymbol{R}$ are equal to zero. Gerschgorin discs (see, e.g., [18]) provide a lower bound on the minimum eigenvalue

$$
\lambda_{\min } \geq \min _{i}\left\{2 N-\rho_{i}\right\}
$$

where

$$
\rho_{i}=\sum_{j \neq i}\left|\rho_{i j}\right|
$$

is the absolute sum of the off-diagonal elements of the $i$ th row/column of $\boldsymbol{R} . \rho_{i}$ is the radius and $2 N$ is the center of the $i$ th Gerschgorin disc.

Instead of directly minimizing $\operatorname{tr}\left\{R^{-1}\right\}$ we suggest minimizing the largest absolute sum $\rho_{\max }=\max _{i}\left\{\rho_{i}\right\}$. Intuitively, minimization of the maximum Gerschgorin disc radius attempts a reduction in the eigenvalue spread and forces the matrix $\boldsymbol{R}$ to have a form, which is as close as possible to the diagonal form. The sequence pair that minimizes the merit factor $\rho_{\max }$ is called the optimal pair. The two-sided pilot-symbol matrix corresponding to the optimal sequence pair is

$$
\hat{\boldsymbol{P}}=\arg \min _{\boldsymbol{P}} \rho_{\max }
$$

where $\boldsymbol{P}$ has the form given in (1).

When $\rho_{\max }=0$ the Grammian matrix $\boldsymbol{R}$ is the diagonal matrix $2 N \cdot I$, where $I$ is the identity matrix. The ML channel estimation based on the corresponding sequence pair achieves the absolute minimum variance lower bound $\frac{N_{0}}{E_{s}} \frac{L}{N}$. In the next section, we will suggest sequence pairs for even $N$ that achieve $\rho_{\max }=0$. Unfortunately, when $N$ is odd, this equality (and the minimum CRLB) cannot be achieved. Nevertheless, for a subset of odd $N$ we will show how to construct sequence pairs that achieve the minimum possible merit factor $\rho_{\max }=2\left\lfloor\frac{L-1}{2}\right\rfloor$. In addition to optimal sequences, we also define as "good" sequence pairs those pairs for which $\rho_{\max }<2 N$. Note that this condition on $\rho_{\max }$ assures that $\lambda_{\min }>0$ and, consequently, that the CRLB is bounded.

\section{SEQuence Design}

Given a sequence $\boldsymbol{u}$ of length $N$, we define its even- and oddperiodic extensions as, respectively, $\boldsymbol{u}^{e}$ and $\boldsymbol{u}^{\circ}$. The (periodic) autocorrelation of $\boldsymbol{x}$ is defined as $R^{\boldsymbol{x}}(l)=\sum_{k=0}^{N-1} x_{k} x_{k+l}$. In the following, the period $N$ will be implied in all definitions of the form $R^{x}(l)$, regardless of the periodicity of $x$. In a similar manner, the aperiodic autocorrelation function of a sequence $\boldsymbol{u}$ is defined as $R_{a p}^{u}(l)=\sum_{k=0}^{N-l-1} u_{k} \cdot u_{k+l}$, for $0 \leq l \leq N-1$, $R_{a p}^{u}(l)=R_{a p}^{u}(-l)$, for $-N+1 \leq l<0$ and zero, otherwise.

First, we investigate pilot-symbol sequence pairs that achieve $\rho_{\max }=0$, i.e., $\rho_{i j}=0$ for all $i \neq j$. From (2), we see that $\rho_{\max }=0$ iff

$$
\begin{aligned}
\mathbf{0} & =\left[\boldsymbol{P}_{1}^{H} \boldsymbol{P}_{1}\right]_{i j}+\left[\boldsymbol{P}_{2}^{H} \boldsymbol{P}_{2}\right]_{i j} \\
& =\left[\boldsymbol{P}_{1}\right]_{i}^{H}\left[\boldsymbol{P}_{1}\right]_{j}+\left[\boldsymbol{P}_{2}\right]_{i}^{H}\left[\boldsymbol{P}_{2}\right]_{j}
\end{aligned}
$$

for all $i \neq j$. Here, $\left[\boldsymbol{P}_{1}\right]_{i j}$ and $\left[\boldsymbol{P}_{1}\right]_{i}$, denote, respectively, the $(i, j)$ th element and the $i$ th column vector of $\boldsymbol{P}_{1}$. Same notation applies to any other matrix.

Since $\left[\boldsymbol{P}_{1}\right]_{i}$ is a snapshot of a shifted preamble block it is easy to see that, for an arbitrary (i.e., not necessarily periodic) pilot-symbol sequence, the term $\left[\boldsymbol{P}_{1}\right]_{i}^{H}\left[\boldsymbol{P}_{1}\right]_{j}$ can be considered as a partial correlation of a sequence whose period is larger then $N+L-1$. Here, we are considering only a snapshot of length $N+L-1$ of either an even- or an odd-periodic extension of a basic sequence $\boldsymbol{p}_{1}$ of length $N$ to be used as a pilot-symbol block. For such a pilot-symbol sequence the term $\left[\boldsymbol{P}_{1}\right]_{i}^{H}\left[\boldsymbol{P}_{1}\right]_{j}$ is equal to either $R^{\boldsymbol{p}_{1}^{e}}(i-j)$ or $R^{\boldsymbol{p}_{1}^{o}}(i-j)$. Clearly, $\left[\boldsymbol{P}_{1}\right]_{i}^{H}\left[\boldsymbol{P}_{1}\right]_{j}$ is only a function of the difference $i-j$. The corresponding two-sided Grammian matrix $R$ is a Toeplitz matrix.

Periodic sequence pairs whose autocorrelation values at nonzero shifts add to a zero are called periodic complementary sequences. An example of such sequences are Golay complementary sequences [14]. Golay complementary sequences satisfy even a stronger condition: the aperiodic autocorrelation of a pair of complementary Golay sequences adds up to a zero for nonzero shifts. Complementary Golay sequences exist only for periods $N=2^{a} \cdot 10^{b} \cdot 26^{c}$, where $a, b$, and $c$ are nonnegative integers. Fortunately, Luke [15] has introduced odd-periodic complementary sequences that exist for most even $N$ smaller than 50 (except $N=38$ and $N=46$ ). Therefore, for most even $N<50$ we can achieve $\rho_{\max }=0$ and the minimum possible CRLB of $\frac{N_{0}}{E_{s}} \frac{L}{2 N}$.

Next, we introduce almost-complementary periodic sequences with an odd period. These sequences achieve the minimum possible $\rho_{\max }$ for odd $N$. Before presenting these sequences we first introduce several lemmas and theorems. They prove (among other facts) that there are no periodic complementary sequences with an odd period and that the minimum achievable $\rho_{\max }$ is $2\left\lfloor\frac{L-1}{2}\right\rfloor$ when $N$ is odd. They 
also lead to the construction of sequences that achieve this minimum.

Lemma 1: No three mutually orthogonal vectors with elements in $\{ \pm 1\}$ of length $2 N$, where $N$ is an odd number, exist. Furthermore, if $\boldsymbol{a}$ and $\boldsymbol{b}$ and $\boldsymbol{b}$ and $\boldsymbol{c}$ are mutually orthogonal, then $\boldsymbol{a}$ and $\boldsymbol{c}$ are either equal or differ in an even number of positions.

Proof: Two vectors with elements in $\{ \pm 1\}$ of length $2 N$ are orthogonal iff they differ in $N$ positions. Let us assume that pairs of vectors $\boldsymbol{a}$ and $\boldsymbol{b}$ and $\boldsymbol{b}$ and $\boldsymbol{c}$ are mutually orthogonal. It is easy to see that $\boldsymbol{a}$ and $\boldsymbol{c}$ can be equal or can differ in $2,4, \ldots, N-1, N+1, \ldots, 2 N$ positions. That is, they have to differ in an even number of positions. Since in this case $N$ is an odd number, they cannot be orthogonal.

Theorem 1: Let $\boldsymbol{M}$ be a square $L \times L$ Grammian matrix $\boldsymbol{M}=\boldsymbol{S}^{H} \boldsymbol{S}$, where column vectors of $\boldsymbol{S}$ are of length $2 N, N$ is an odd number, and the elements of $\boldsymbol{S}$ are in $\{ \pm 1\}$. There is at least one row/column of $\boldsymbol{M}$ with the number of zeros less than $\left\lceil\frac{L+1}{2}\right\rceil$.

Proof: First, we will demonstrate the proof for odd $L$. The proof is aided with Figs. 2 and 3. Clearly, the diagonal elements of $M$ are all equal to $2 N$. A diagonal element is denoted with a $\bullet$ in Figs. 2 and 3. If there is a column/row that has no elements equal to zero then the theorem is satisfied. Let us assume that each column/row has at least one zero and that the zeros are distributed as shown in Fig. 2. If they are not distributed in the shown way, we can arrive to this form by permuting the rows/columns of the matrix. A permutation does not change the number of zeros per row/column, it only changes their positions. Note that, since $L$ is an odd number, there has to be at least one row/column that holds two zeros.

Lemma 1 says that no three binary vectors of length $2 N$ (where $N$ is odd) can be mutually orthogonal. It is easy to see that this constraint implies that no rectangle such that one of its corners is a diagonal element, can have zeros on all of its other three corners, as shown in Fig. 3. " $X$ " in this figure denotes a nonzero element. Shaded " 0 " and " $X$ " denote possible location of zero and nonzero elements of $\boldsymbol{M}$. Lemma 1 implies that element $[\boldsymbol{M}]_{1,3}$ has to be nonzero and this element is not shaded. Furthermore, only one of the elements $[\boldsymbol{M}]_{1,4}$ and $[\boldsymbol{M}]_{1,5}$ can be zero. The same applies to the pairs of elements

$\left([\boldsymbol{M}]_{1,6},[\boldsymbol{M}]_{1,7}\right),\left([\boldsymbol{M}]_{1,8},[\boldsymbol{M}]_{1,9}\right), \ldots,\left([\boldsymbol{M}]_{1, L-1},[\boldsymbol{M}]_{1, L}\right)$.

Clearly, the number of zeros in the first row cannot be larger than $1+(L-3) / 2=(L-1) / 2<(L+1) / 2$.

For even $L$, we can use the same approach to show that there cannot be a row with more than $L / 2$ zeros. Since the matrix $\boldsymbol{M}$ is symmetric, the same arguments apply to columns of $M$.

Corollary 1: There are no even- and odd-periodic complementary sequences with respective fundamental periods $N$ and $2 N$, where $N$ is odd.

Theorem 2: Let $\boldsymbol{u}$ and $\boldsymbol{v}$ be two sequences of length $N$, where $N$ is an odd number, related to each other as follows: $v_{i}=$ $(-1)^{i} u_{i}$ for $0 \leq i \leq N-1$. Then

$$
R^{\boldsymbol{v}^{e}}(l)+R^{\boldsymbol{u}^{o}}(l)=\left[1+(-1)^{l}\right] R^{\boldsymbol{v}^{e}}(l) .
$$

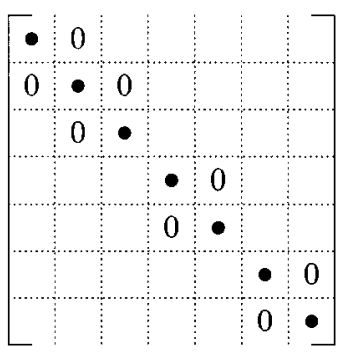

Fig. 2. Locations of single zeros per column/row after permutations.

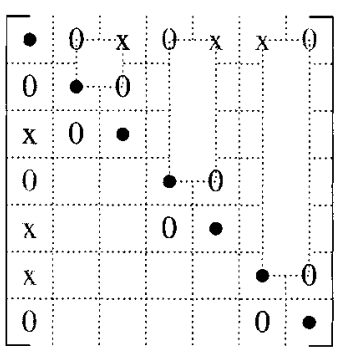

Fig. 3. Possible location of zeros in the first column/row while satisfying the conditions of Lemma 1

Proof: First, we state two known results (see, e.g., [19]). The autocorrelation functions of even- and odd-periodic extensions of $u$ (and $v$ ) are related to the aperiodic autocorrelation function of $\boldsymbol{u}$ as follows: $R^{\boldsymbol{u}^{e}}(l)=R_{a p}^{\boldsymbol{u}}(l)+R_{a p}^{\boldsymbol{u}}(N-|l|)$ and $R^{\boldsymbol{u}^{e}}(l)=R_{a p}^{\boldsymbol{u}}(l)-R_{a p}^{\boldsymbol{u}}(N-|l|)$ for $0 \leq|l| \leq N-1$.

The aperiodic autocorrelation functions of $\boldsymbol{u}$ and $\boldsymbol{v}$ are related as follows: $R_{a p}^{\boldsymbol{v}}(l)=(-1)^{l} R_{a p}^{\boldsymbol{u}}(l)$ for all shifts $l$.

It follows that

$$
\begin{aligned}
R^{\boldsymbol{v}^{e}}(l) & =R_{a p}^{\boldsymbol{v}}(l)+R_{a p}^{\boldsymbol{v}}(N-l) \\
& =(-1)^{l} R_{a p}^{\boldsymbol{u}}(l)+(-1)^{|N-l|} R_{a p}^{\boldsymbol{u}}(N-l) \\
& =(-1)^{l}\left[R_{a p}^{\boldsymbol{u}}(l)-R_{a p}^{\boldsymbol{u}}(N-l)\right] \\
& =(-1)^{l} R^{\boldsymbol{u}^{o}}(l), \quad 0 \leq|l| \leq N-1
\end{aligned}
$$

since $N$ in odd. Equation (4) follows when we observe that $R^{\boldsymbol{u}^{e}}(l)$ and $R^{\boldsymbol{u}^{o}}(l)$ have respective periods $N$ and $2 N$.

Corollary 2: Theorems 1 and 2 imply that sequences having an odd period $N$ with the property $\left|R^{\boldsymbol{u}^{e}}(l)\right|=1$ for $1 \leq l \leq$ $N-1$ allow for construction of almost-complementary periodic sequences that achieve $\min _{\boldsymbol{P}} \rho_{\max }=2\left\lfloor\frac{L-1}{2}\right\rfloor$. The minimization is taken over all sequence pairs not necessarily periodic.

Periodic extensions of Barker sequences having odd lengths $3,5,7,11$, and 13 , and $m$-sequences of periods $N=2^{m}-1$ satisfy this property. Other sequences for which $\left|R^{\boldsymbol{u}^{o}}(l)\right|=1$ can be derived based on cyclic difference sets. These sequences include (see, e.g., [20]) quadratic residue (or Legendre) sequences of period $p=(3 \bmod 4)$, where $p$ is a prime, and twin-prime sequences of period $p(p+2)$, where $p$ is also a prime. Therefore, for odd

$$
N \in\{3,5,7,11,13,15,19,23,31,35,43,47\}
$$

we can design almost-complementary periodic sequence pairs. Snapshots of almost-complementary sequences of length $N+$ $L-1$ can be used as pilot symbol blocks minimizing the merit factor $\rho_{\max }$. 
TABLE I

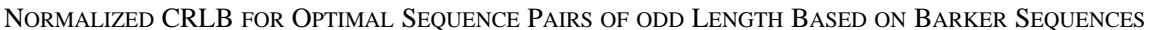

\begin{tabular}{|c|c|c|c|c|c|}
\hline$N$ & 3 & 5 & 7 & 11 & 13 \\
\hline Normalized CRLB & 0.5417 & 0.5298 & 0.5375 & 0.5298 & 0.5124 \\
\hline Increase Relative to 0.5 in dB & 0.3479 & 0.2514 & 0.3141 & 0.2414 & 0.1064 \\
\hline
\end{tabular}

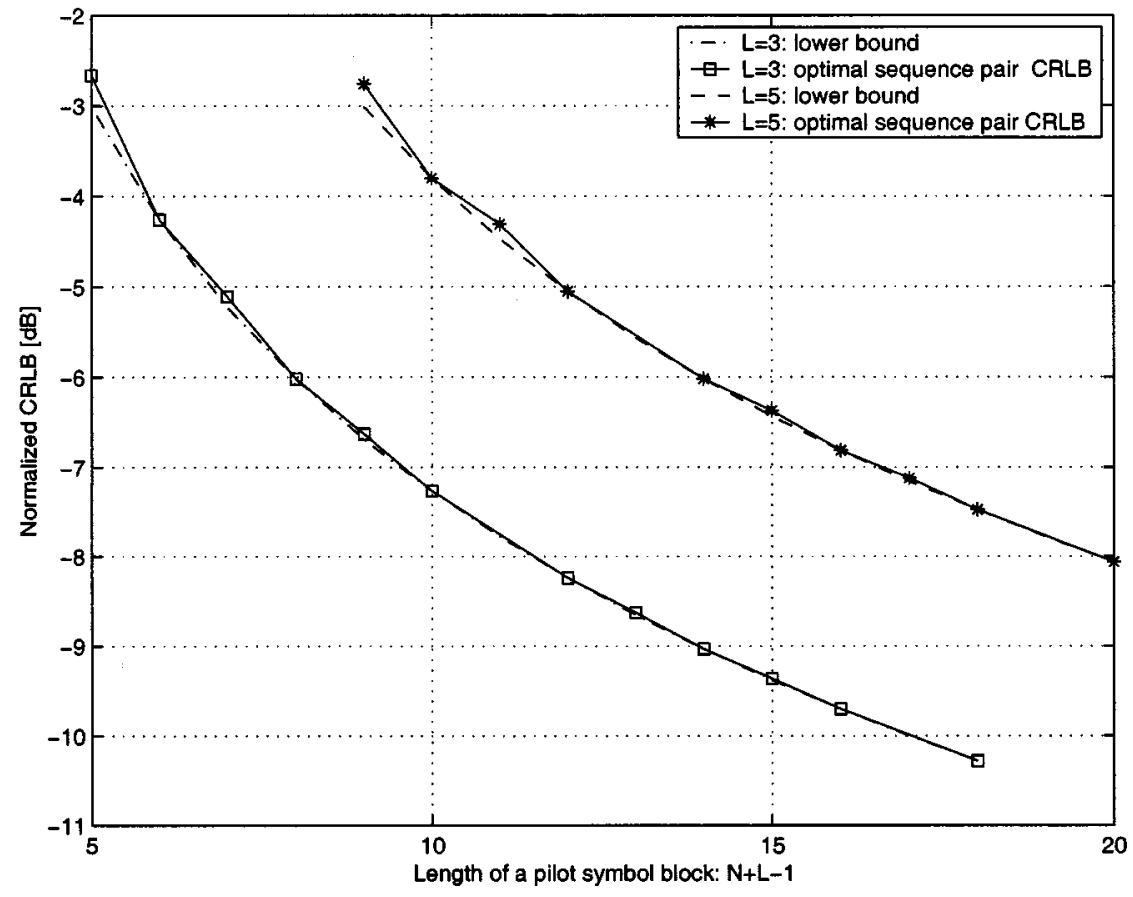

Fig. 4. Normalized CRLB for optimal sequence pairs and channel lengths $L=3$ and $L=5$.

For a subset of $N$ prime and $N=1 \bmod 4$ (for $N \in$ $\{17,29,37,49\}$ ), sequences that have half of their PACF values equal to 1 and the other half equal to -3 exist. Their construction is given in [21]. The ensuing sequence pairs evaluate the merit factor to $4\lfloor(L-1) / 2\rfloor$. Note that by using such sequences we can generate "good" sequence pairs defined earlier as the pairs for which $\rho_{\max }<2 N$. This condition guarantees that the two-sided Grammian $\boldsymbol{R}$ is positive definite and that the ML estimation variance is bounded.

\section{ANALYSIS}

It is clear that the complementary sequence pairs that minimize $\rho_{\max }$ for even $N$ also minimize the normalized ML estimation variance $\operatorname{tr}\left(\boldsymbol{R}^{-1}\right)$. However, it is not clear whether the optimal sequence pairs for odd $N$ also minimize the ML estimation variance. In a limited attempt to determine an answer, we have compared the CRLB for two sequence pairs constructed based on Barker sequences of lengths $N=3$ and $N=5$ to arbitrary pairs of sequences of the same length using an exhaustive computer search. The results for $N=L$, indicate that the same minimum possible normalized variances of 0.5417 for $N=3$ and 0.5298 for $N=5$ are achieved with the constructed sequence pairs. Normalized CRLBs for sequence pairs based on all Barker sequences of odd lengths for $N=L$ are given in Table I. For even $N$, the constructed sequences have a normalized CRLB of 0.5 . It is clear that the CRLBs of the constructed sequences for odd $N$ are slightly larger than 0.5 (within $0.35 \mathrm{~dB}$ ). We have left as an open problem whether other optimal sequence pairs introduced in terms of the mini-max criterion also allow for a close to 0.5 normalized estimation CRLB.

Fig. 4 demonstrates the impact of an increase in the pilotsymbol block length for channels of lengths three and five on the normalized ML estimation variance in decibels for the constructed optimal sequence pairs. We can see that an increase in the number of pilot symbols by three for $L=3$ and by four for $L=5$ allows for a reduction in the estimation variance by approximately $3 \mathrm{~dB}$. From this plot it is again clear that constructed optimal sequence pairs for odd $N$ allow for close to minimal ML estimation variance.

In order to determine the significance of the two-sided optimal sequence design for data detection we study the performance of a ML receiver that assumes that the channel estimate obtained based on the two-sided pilot-symbol sequences is the true channel. Detection performance is studied for two cases. In the first case, the length $L=3$ channel impulse response $\boldsymbol{h}$ is an independent and identically distributed (i.i.d.) complex Gaussian vector having a different realization at each simulated block. The results in this case assess the error-probability performance of the pilot sequences, averaged over a large number 


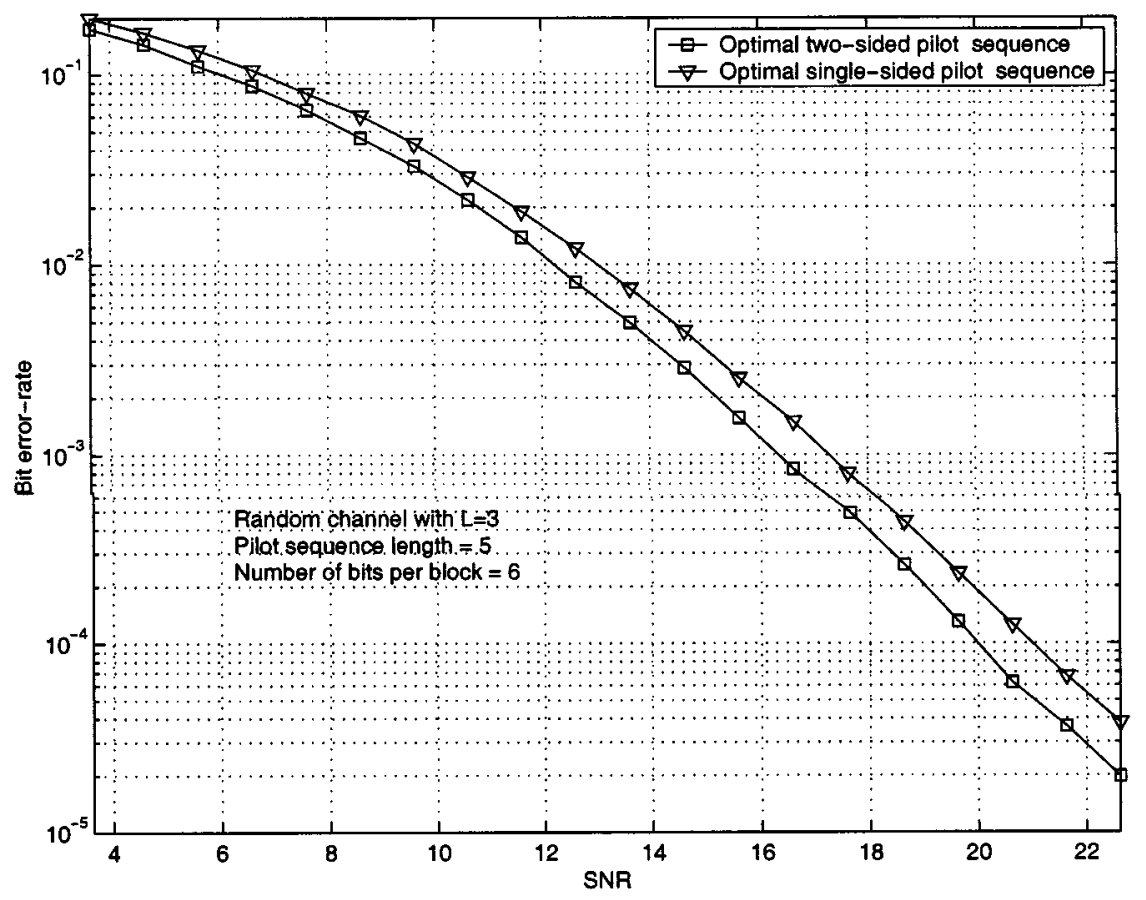

Fig. 5. ML sequence detection performance based on optimal two-sided and optimal one-sided pilot symbol sequence pairs: random channel.

of ISI channels. The second case analyzes the maximum-likelihood sequence estimation (MLSE) performance for a real (and fixed) channel response $h=\left[\begin{array}{lll}0.407 & 0.815 & 0.407\end{array}\right]^{T}$ with spectral nulls for a set of frequencies (see [22]).

We analyze the bit-error-rate performance for two two-sided pilot sequence pairs with sequences of length 5 and $D=6$. The first pair is optimally designed as per Section IV:

$$
\text { ([1, 1, -1, 1, 1], [1, -1, -1, -1, 1]). }
$$

The corresponding pair of autocorrelation functions have values in $(\{3,-1,-1\},\{3,1,-1\})$ and their sum has values in $\{6,0,-2\}$. The second pair consists of two equal sequences

$$
([1,1,-1,1,1],[1,1,-1,1,1])
$$

where $[1,1,-1,1,1]$ minimizes the CRLB of the one-sided ML channel estimate. The sum of autocorrelation functions for these two sequences has values in $\{6,-2,-2\}$. Normalized estimation variances are equal to 0.5417 and 0.75 (a gain of 1.4 $\mathrm{dB}$ ) for, respectively, the optimal two-sided and the optimal onesided sequence pairs. Fig. 5 shows that for the random channel the performance degradation due to the use of an optimal singlesided pilot sequence is above $1 \mathrm{~dB}$ at an error-rate of $10^{-4}$ and lower. On the other hand, from Fig. 6 we see that the increased estimation variance for a fixed real channel can cause a performance degradation of more than $1 \mathrm{~dB}$ for error rates lower than $10^{-3}$.

For a fixed $E_{b} / N_{0}$ ( $E_{b}$ is the energy per information bit related to $E_{s}$ as $E_{b}=E_{s} D /(D+N+L-1)$ ) and given $D$ (possibly determined to ensure the quasi-static property of channel coefficients and/or based on complexity considerations) and $L$, an optimal $N$ exists. This is due to the fact that an increase in $N$ reduces the estimation variance and, thus, allows for a decrease in the detection error. On the other hand, given that the per information bit energy is fixed, an increase in the number of transmitted information symbols $D$ decreases the symbol energy $E_{s}$, which affects both the estimation variance and the detection error rate. Of course, an increase in the number of pilot symbols also expands the required bandwidth for a given information rate. To illustrate the existance of an optimal $N$ we have plotted in Fig. 7 the signal-to-noise ratio (SNR) loss relative to the known channel (nonpilot symbol aided) case at the bit error rate of $10^{-5}$ versus $N$. The results are given for the fixed channel response $h=\left[\begin{array}{lll}0.407 & 0.815 & 0.407\end{array}\right]^{T}, D=6$, and the sequence pair that minimizes the merit factor $\rho_{\max }$ at each $N$. It is clear, that the optimal generating sequence length is $N=4$ in which case the optimal two-sided sequence pair is

$$
([1,1,-1,1,1,1],[1,1,-1,1,1,1]) \text {. }
$$

That is, in this special case a periodic insertion of a single sequence optimal for single-sided channel estimation is also optimal for two-sided channel estimation. The gain over the $N=3$ case is less than $0.5 \mathrm{~dB}$ and the bandwidth expansion is $9 \%$.

\section{CONCLUSION}

Snapshots of periodic complementary sequence pairs are optimal for two-sided ISI channel estimation for even $N \geq L$, where $(N+L-1)$ is the length of a pilot-symbol block and $L$ is the number of channel taps. These sequence pairs allow for the minimum possible estimation variance $\frac{N_{0}}{E_{\mathrm{s}}} \frac{L}{2 N}$ for channel estimation that attains the CRLB. The minimum possible variance, $\frac{N_{0}}{E_{s}} \frac{L}{N}$, of the "one-sided" channel estimator is attainable when either $L \leq 4$ and $N \in\{1,2,0 \bmod 4\}$ or $N=2^{L}+L$ (see [8]).

No complementary sequences exist for odd $N$ and, therefore, the minimum ML estimation variance is larger than $\frac{N_{0}}{E_{s}} \frac{L}{2 N}$ for 


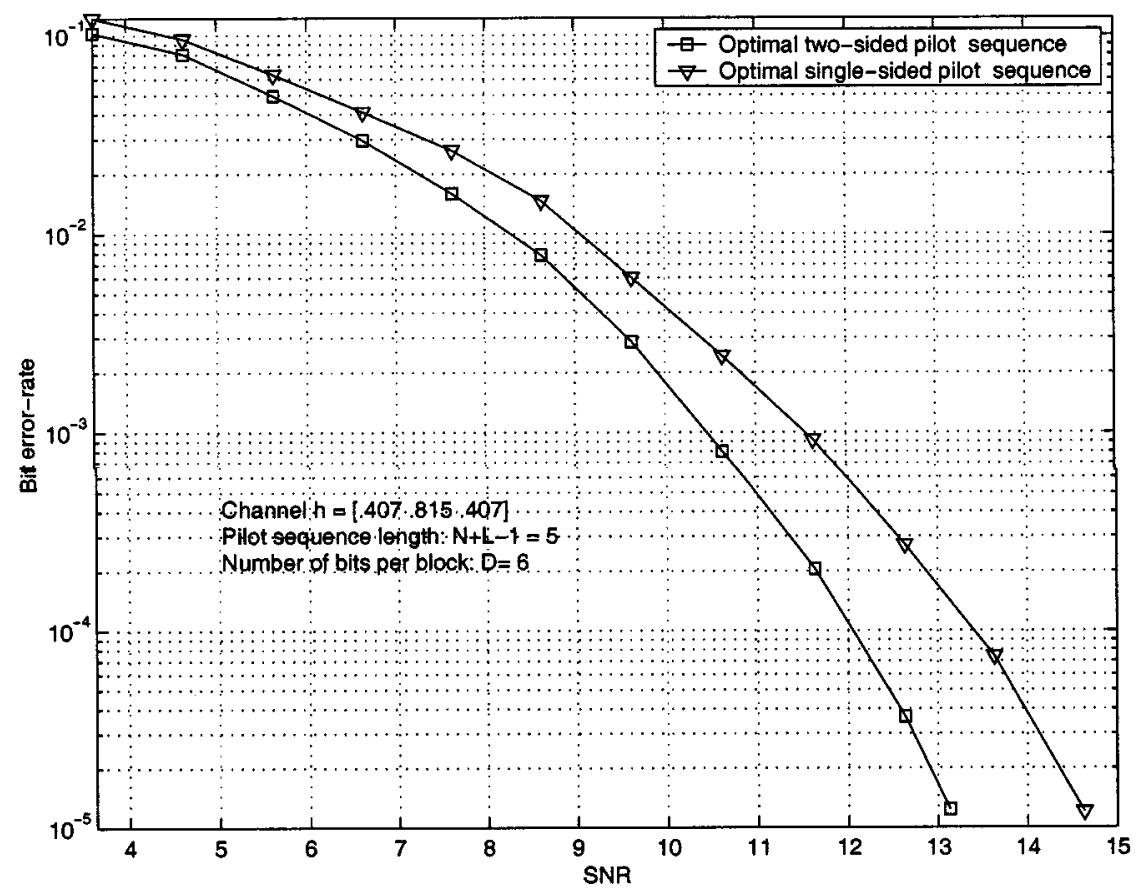

Fig. 6. ML sequence detection performance based on optimal two-sided and optimal one-sided pilot symbol sequence pairs: fixed channel.

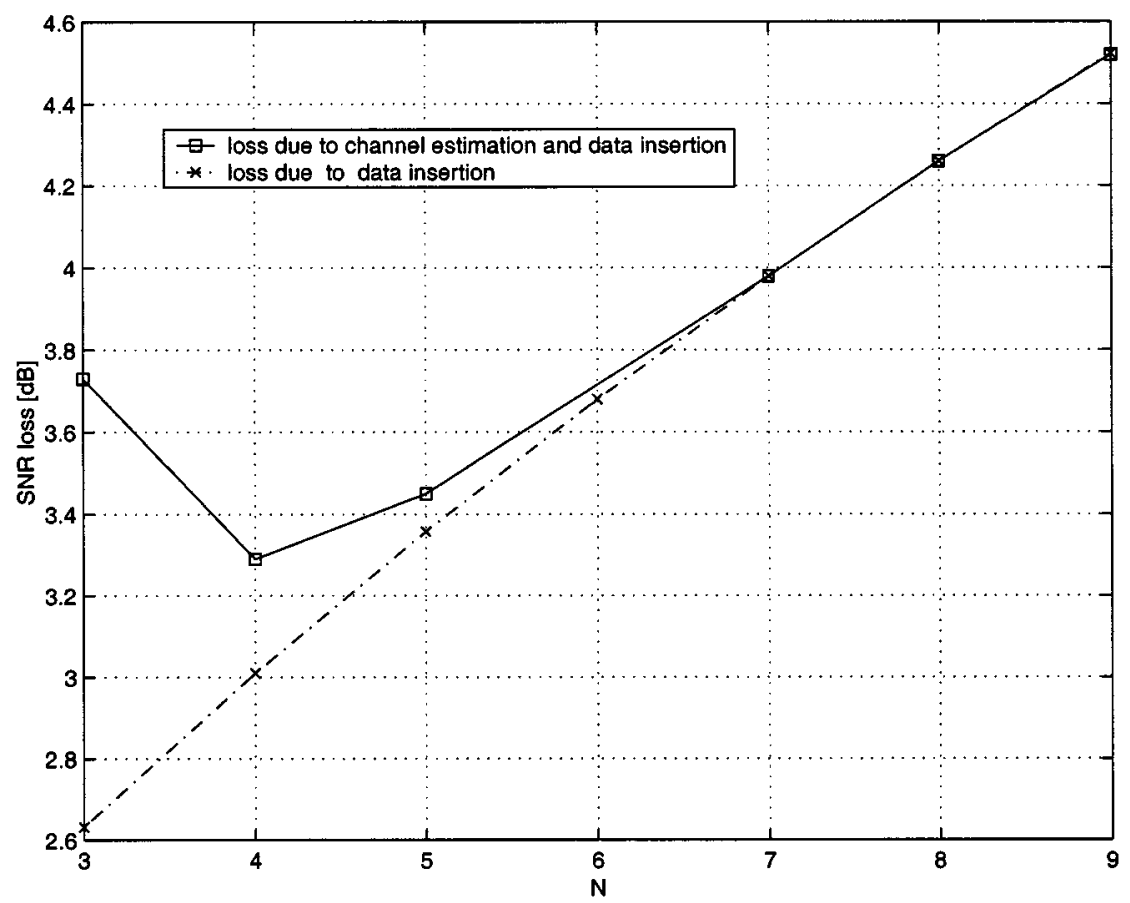

Fig. 7. ML sequence detection $E_{b} / N_{0}$ loss for the symbol error rate of $10^{-5}$ due to the two-sided channel estimation error and/or pilot sequence insertion relative to the case of a known channel and no pilot sequence insertion: fixed channel.

odd $N$. An upper bound on the estimation CRLB is based on the Gerschgorin discs of the matrix formed by summing the autocorrelation matrices of the two training sequences that frame a data block. This upper bound can be minimized by minimizing $\rho_{\max }=\max _{i} \sum_{j \neq i}\left|\rho_{i j}\right|$ over all sequence pairs. For even $N$, periodic complementary sequences achieve the minimum pos- sible merit factor $\rho_{\max }=0$. Periodic sequence pairs that minimize the merit factor $\rho_{\max }$ have been constructed for a subset of odd $N$. We have shown that these pairs achieve the minimum possible $\rho_{\max }=2\lfloor(L-1) / 2\rfloor$ for odd $N$. The pairs with odd $N$ that achieve $\rho_{\max }$ have been termed almost-complementary sequences. 
For two constructed almost-complementary sequences we have shown that they allow for channel estimation variance that is slightly larger than $\frac{N_{0}}{E_{S}} \frac{L}{2 N}$ and equal to the minimum possible variance for given pilot-symbol block lengths.

Simulations have demonstrated that a significant detection performance improvement can be achieved when optimal pilot symbol sequence pairs are used for given parameters $N, D$, and $L$. Furthermore, an optimization over $N$ which neglects the effect of bandwidth expansion can provide an additional performance gain.

\section{REFERENCES}

[1] J. K. Cavers, "An analysis of pilot symbol assisted modulation for Rayleigh fading channels," IEEE Trans. Veh. Technol., vol. 40, pp. 686-693, Nov. 1991.

[2] S. N. Crozier, D. D. Falconer, and S. A. Mahmoud, "Reduced complexity short-block data detection techniques for fading time-dispersive channels," IEEE Trans. Veh. Technol., vol. 41, pp. 255-265, Aug. 1992.

[3] H. Meyr, M. Moenenclaey, and S. A. Fechtel, Digital Communication Receivers. New York: Wiley, 1997.

[4] C. N. Georghiades and J. Han, "Sequence estimation in the presence of random parameters via the EM algorithm," IEEE Trans. Commun., vol. 45, pp. 300-308, Mar. 1997.

[5] K.-H. Chang, W. S. Yuan, and C. N. Georghiades, "Block-by-block channel and sequence estimation for ISI/fading channels," in Signal Processing in Telecommunications. London, U.K.: Springer-Verlag, 1995.

[6] J. Salz, "On the start-up problem in digital echo cancelers," Bell Syst. Tech. J., vol. 62, pp. 1353-1364, July-Aug. 1983.

[7] A. Milewski, "Periodic sequences with optimal properties for channel estimation and fast start-up equalization," IBM J. Res. Develop., vol. 27, no. 5 , pp. 426-431, Sept. 1983.
[8] S. N. Crozier, D. D. Falconer, and S. A. Mahmoud, "Least sum of squared errors (LSSE) channel estimation," Proc. Inst. Elect. Eng., pt. F, vol. 138, pp. 371-378, Aug. 1991.

[9] K.-H. Chang, "Joint maximum-likelihood sequence and channel estimation for intersymbol interference channels," Ph.D. dissertation, Texas A \& M Univ., College Station, 1995.

[10] C. Tellambura, M. G. Parker, Y. J. Guo, S. J. Shepherd, and S. K. Barton, "Optimal sequences for channel estimation using discrete Fourier techniques," IEEE Trans. Commun., vol. 47, pp. 230-238, Feb. 1999.

[11] L. Bomer and M. Antweiler, "Binary and biphase sequences and arrays with low periodic autocorrelation sidelobes," in Proc. ICASSP'90, Apr. 1990 , pp. $1663-1666$.

[12] C. N. Georghiades, "On PPM sequences with good autocorrelation properties," IEEE Trans. Inform. Theory, vol. 34, pp. 571-576, May 1988.

[13] C. Tellambura, "Phase optimization criterion for reducing peak-to-average power ratio in OFDM," Electron. Lett., vol. 34, pp. 169-170, Jan. 1998.

[14] M. J. E. Golay, "Complementary series," IEEE Trans. Inform. Theory, vol. IT-7, pp. 82-87, Apr. 1961.

[15] H. D. Luke, "Binary odd-periodic complementary sequences," IEEE Trans. Inform. Theory, vol. 43, pp. 365-367, Jan. 1997.

[16] S. Grob and P. Clark, "Enhanced channel impulse response identification for the ITU HF measurement campaign," Electron. Lett., vol. 34, pp. 1022-1023, May 1998.

[17] C. Tellambura, Y. Guo, and S. Barton, "Channel estimation using aperiodic binary sequences," Commun. Lett., vol. 2, pp. 140-142, May 1998.

[18] R. Horn and C. Johnson, Matrix Analysis. Cambridge: Cambridge Univ. Press, 1996.

[19] D. Sarwate and M. Pursley, "Cross-correlation properties of pseudorandom and related sequences," Proc. IEEE, vol. 68, pp. 593-619, May 1980.

[20] S. W. Golomb, Shift Register Sequences. Laguna Hills, CA: Aegean, 1982 .

[21] A. M. Boehmer, "Binary pulse compression codes," IEEE Trans. Inform. Theory, vol. IT-13, pp. 156-167, Apr. 1967.

[22] J. G. Proakis, Digital Communications. New York: McGraw-Hill, 1989. 